\title{
Response-to-stimulus interval does not affect implicit motor sequence learning, but does affect performance
}

\author{
DANIEL B. WILLINGHAM, ANDREW R. GREENBERG, and R. CANNON THOMAS \\ University of Virginia, Charlottesville, Virginia
}

\begin{abstract}
Nissen and Bullemer (1987) reported that implicit motor sequence learning was disrupted by the addition of a secondary task. They suggested that this effect was due to the attentional load that the secondary task adds. Recently it has been suggested that the attentional load is not critical, but rather that the secondary task affects timing, either by lengthening or by making inconsistent the response-tostimulus interval (RSI) - that is, the delay between when a subject makes a response and when the next stimulus appears. In six experiments we manipulated the RSI and found no support for these two hypotheses. An inconsistent RSI did not adversely affect implicit motor sequence learning. A long RSI did not affect learning, although under some conditions subjects did not express learning if the RSI was long. These results are interpreted as reflecting the effects of attention.
\end{abstract}

Much effort during the past decade has gone toward demonstrating that implicit and explicit memory represent the workings of separate neural and cognitive systems (see Schacter, Chiu, \& Ochsner, 1993; Squire, Knowlton, \& Musen, 1993, for reviews). Implicit memory is tested via performance, without direct reference to the encoding episode, and need not be associated with awareness on the part of the learner that he or she has learned. Explicit memory tests do make direct reference to the encoding episode; the learner is aware that learning has taken place and is conscious of recalling the material (Graf \& Schacter, 1985).

Effort has gone not only into demonstrating that these two types of memory are different in kind, but also toward characterizing them. It is well established that explicit memory requires attention to the to-be-remembered material at encoding (see, e.g., Glucksberg \& Cowan, 1970). The relationship of attention to implicit memory has not been quite so clear.

A number of implicit-memory paradigms have been used to investigate the role of attention and implicit memory. The one addressed here involves a perceptual motor skill: serial response time (SRT) learning. In the SRT task, the subject is presented with a four-choice RT task. Typically the stimuli are spatial positions, arrayed horizontally, and the subject uses the index and middle finger of each hand to respond via keypresses. The subject is not told that the stimuli appear in a repeating sequence, which is usually 12 units long. Because nothing marks the beginning or end of the sequence, many subjects do not notice that the stimuli are sequenced. Never-

The authors thank Peter Frensch, Steve Keele, and Geoff Loftus for many helpful suggestions on an earlier version of this manuscript. Correspondence concerning this article should be addressed to D. B. Willingham, Department of Psychology, University of Virginia, 102 Gilmer Hall, Charlottesville, VA 22903 (e-mail: dbw8m@virginia.edu). theless, their pattern of RTs shows that they have learned it; they respond faster and faster in the presence of the sequence, and their responses slow if the stimuli appear randomly (Nissen \& Bullemer, 1987; Willingham, Nissen, \& Bullemer, 1989)

Several experiments have shown that this learning is retarded if subjects are asked to perform a secondary task along with the SRT task. Experimenters have used tone counting for this purpose. In the tone-counting task, subjects hear a high- or a low-pitched tone on each trial, and they must count the number of low-pitched tones and report the total at the end of each trial block. Nissen and Bullemer (1987) reported that learning was virtually eliminated when the secondary task was added, a finding replicated by other experimenters (Keele \& Jennings, 1992), although the effect may not be obtained for certain classes of sequences (A. Cohen, Ivry, \& Keele, 1990; Curran \& Keele, 1993)

Such researchers have assumed that the effect of the tone-counting task is to occupy attentional resources. Recently, however, Frensch and Miner (1994) and Stadler (1995) have pointed out that other interpretations are possible. Frensch and Miner argue that another effect of the tone-counting task is to lengthen the response-tostimulus interval (RSI). The secondary task inevitably takes some time to perform, so even if the stimulus is present on the screen, if the subject is performing the secondary task, the effective RSI is lengthened. Frensch and Miner argue that successive units in the sequence must occupy working memory simultaneously for sequence learning to occur, and that the long RSI allows one unit to fade from working memory before the next unit is presented. Frensch and Miner reported that sequence learning is retarded if a 1,500 -msec RSI is used.

Stadler (1995) has emphasized the variability in RSI that results from the tone-counting task. He points out 
that certain processes associated with the secondary task must be performed on every trial (e.g., categorizing the tone), but other processes are performed on only half of the trials-specifically, incrementing the running count of target tones. Stadler (1995) argues that some trials have a longer effective RSI than others do for this reason, and that the secondary task impedes sequence learning because of the inconsistency of the RSIs. He argues that sequence learning occurs, in part, through organization of the sequence into chunks. Stadler (1993) has shown that this organization can be facilitated if the experimenter inserts pauses in consistent locations within the sequence and that it can be made more difficult if the pauses are inserted in random places. Stadler (1995) argues that the tone-counting task makes the effective RSI long on half of the trials and short on the other half, thereby disrupting the organization. In an attempt to approximate the effective RSIs in the tone-counting task, Stadler (1995) reported that subjects showed impaired sequence learning when half the trials had a 2,000-msec RSI and half had a 500-msec RSI.

The purpose of the work reported here is to examine these alternative accounts of the tone-counting task more closely. Neither Frensch and Miner (1994) nor Stadler (1995) used transfer conditions to test whether subjects trained with inconsistent or with long RSIs would show normal learning with a short, consistent RSI. In other words, the impaired learning that they reported may have been a performance effect, not a learning effect. A second goal was to test Stadler's hypothesis that inconsistent RSIs impair sequence learning, but to do so by using short RSIs. Stadler's use of 500-msec and 2,000-msec RSls unfortunately makes it difficult to determine whether the effect that he observed was due to the long RSI or to the inconsistency of the two RSIs.

\section{EXPERIMENT 1}

The purpose of experiment 1 was to evaluate the effect of a short but inconsistent RSI on sequence learning in the SRT task.

\section{Method}

Subjects. Twenty-four undergraduate students at the University of Virginia (12 male) participated in return for course credit in an introductory psychology course.

Stimuli and Apparatus. The experiment was controlled by a Macintosh IIsi computer. Timing was accurate within a tolerance of $2 \mathrm{msec}$. All stimuli were presented in black on a white background on an Apple 14-in. HighRes monitor. Four possible stimulus locations were identified for the subject via four small dots, each $5 \mathrm{~cm}$ from the bottom of the screen and centered horizontally with $2 \mathrm{~cm}$ between each dot. The stimulus on each trial was an ampersand ("\&") approximately $1.5 \mathrm{~cm}$ tall which appeared just below one of the dots. Each of the four locations on the screen was paired with a response key on the Macintosh keyboard: $\mathrm{z}, \mathrm{c}, \mathrm{b}$, and $\mathrm{m}$. Subjects used the index and middle fingers of each hand to respond.

Some trial blocks had sequenced stimuli and some had pseudorandom stimuli (hereafter called random, for the sake of simplicity). For the sequenced stimuli, a corpus of 563 twelve-unit sequences was developed. Within each sequence, the four positions appeared an equal number of times, a stimulus could not follow itself (e.g., 1223), and there could be no runs of four (e.g., 4321) or trills of four (e.g., 1313). Otherwise, the sequences were not constrained. For sequenced trial blocks, 1 of the sequences was selected from the corpus and was appended eight times to form a 96-trial block. The same 12-unit sequence was used to compose the entire sequenced block for a subject. Each subject saw a different repeating sequence, however. For random trial blocks, 8 of the sequences were randomly selected with replacement from the corpus and were appended, with the constraint that the block as a whole had to meet the criteria applied to individual sequences.

Procedure. The subjects were told to rest their fingers lightly on the response keys and to push the corresponding key as quickly as possible when an ampersand appeared. If they pushed an incorrect key, a 120 -msec tone sounded, indicating that they should try again. The ampersand remained visible until the correct response was made. When the subject pressed the correct key, the stimulus was immediately extinguished, and after the RSI, a new stimulus appeared.

The subjects completed eight 96-trial blocks of the SRT task. The subjects were randomly assigned to one of two conditions. For the subjects in the random condition, the RSI was random from trial to trial. The RSIs were 50,450 , and $850 \mathrm{msec}$, and each appeared equally often. For subjects in the consistent condition, the RSI was always $850 \mathrm{msec}$. The consistent RSI condition had only the longest RSI, because longer RSIs might impair learning; in essence, we tried to make the consistent RSI condition, if anything, more difficult than the random RSI condition.

At the end of five trial blocks, the groups switched RSIs: the consistent group saw random RSIs, and the random group saw consistent RSIs.

The first four blocks of trials were sequenced, and the fifth was random. Thus, sequence learning under random or consistent RSI conditions could be evaluated by comparing RTs to the sequenced blocks with RTs to the final random block; to the extent that RTs were slow on the final random block, one could infer that the fast RTs to the sequenced blocks were due, in part, to knowledge of the sequence. For the final three trial blocks of the SRT task, the stimuli were random, then sequenced, and then random. These blocks allowed comparison of RTs to random and to sequenced stimuli under the changed RSI.

After the SRT task was complete, the subjects were asked whether they had noticed that the stimuli were sequenced. They were then told that the stimuli had in fact appeared in a repeating sequence on some of the trial blocks, and that they would now be given a free recall test of their sequence knowledge. The subjects positioned their fingers on the keyboard as they had in the SRT task, and they were asked to reproduce as much of the sequence as they could. Their responses were echoed on the screen, and they pushed the "q" key when they were finished. They were permitted to recall no more than 26 positions.

\section{Results and Discussion}

The subjects were told to respond as quickly as possible without making errors, so accuracy was not expected to be an informative measure. Indeed, accuracy was uniformly high (means of $96 \%-98 \%$ within blocks and conditions), so we will not discuss it further.

The dependent measure of interest is RT. RTs were summarized by finding the median RT for each set of 12 trials and then calculating the mean of the eight medians in each block for each subject. The means across subjects for each condition are shown in Figure 1.

RTs for training and transfer were assessed in separate analyses. Sequence learning can be measured in two ways. First, one can compare the rate at which RTs decrease 


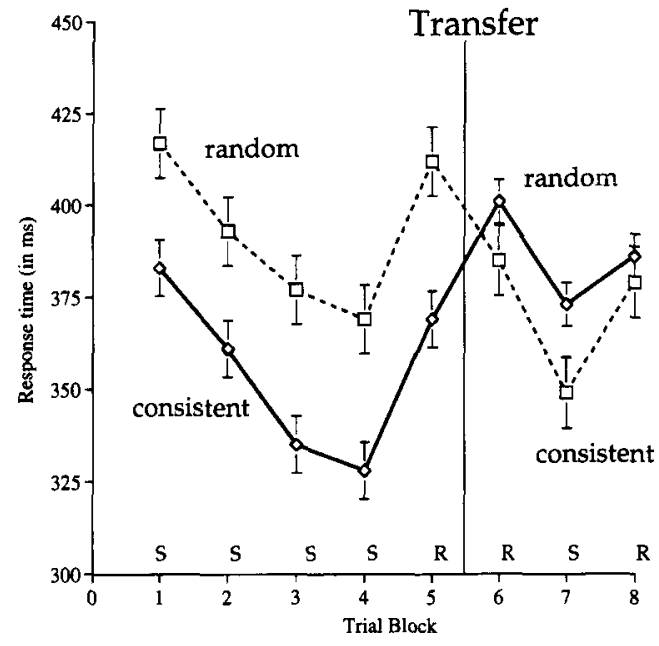

Figure 1. Mean response times across trial blocks as a function of RSI in Experiment 1. Error bars are standard errors, calculated within subjects.

while the stimuli are sequenced (Blocks 1-4). This measure is influenced not only by how well subjects know the sequence, but by improvements in aspects of the task that have nothing to do with the sequence (e.g., learning the stimulus-response mapping). A second measure is the difference in RT between the final sequenced block (Block 4) and the random block (Block 5). This measures the extent to which subjects' RTs increase upon introduction of the random sequence, which may be taken as a relatively pure measure of sequence learning. If subjects know nothing about the sequence, there is no reason why they should respond more slowly on the fifth block when the stimuli begin to appear randomly.

During the four sequenced training blocks, subjects in the random RSI condition responded marginally more slowly than subjects in the consistent condition $[F(1,22)=$ 3.37, $\left.M S_{\mathrm{e}}=9,840, p=.08\right]$. RTs changed reliably across trial blocks $\left[F(3,66)=18.7, M S_{\mathrm{e}}=684, p<.05\right]$, but this change did not vary with condition $[F(3,66)=0.2$, $\left.M S_{\mathrm{e}}=684, p>.20\right]$. A separate analysis of the subjects seeing random RSIs showed that their RTs were, indeed, improving $\left[F(3,33)=7.0, M S_{\mathrm{e}}=768.2, p<.001\right]$.

To assess learning more directly, a difference score was calculated for Blocks 5 and 4 . This measure was reliably different from zero $[t(23)=4.4 ; M=42.8, S E M=$ 9.6], and a comparison across conditions showed no difference $\left[F(1,22)=0.02, M S_{\mathrm{e}}=2,291, p>.20\right]$. When analyzed alone, the random RSI group again showed reliable learning $[t(11)=2.8, p<.01]$.

This pattern was more or less replicated at transfer. To analyze the transfer data, a learning score was derived by subtracting the RT of the sequenced block from the mean of the two random blocks. This measure was significantly different from zero, indicating that subjects showed sequence knowledge at transfer $[t(23)=4.9 ; M=43.3$, $S E M=8.8$, and this knowledge did not differ between conditions $\left[F(1,22)=0.5, M S_{\mathrm{e}}=1,919, p>.20\right]$. When analyzed independently, each group showed reliable learning (both $t \mathrm{~s}>2.0, p \mathrm{~s}<.05$ ).

Explicit knowledge gained was minimal in this experiment and, indeed, all of the experiments reported here. We considered a subject to have explicit knowledge if he or she reported noticing a sequence when queried, or if the subject could correctly produce five or more successive positions of the sequence; these two measures were usually in very good agreement. In Experiment 1, 2 subjects met this criterion, and the data pattern was not qualitatively changed when these subjects were removed from the analysis.

This pattern of explicit performance was replicated in later experiments. No more than 5 subjects gained significant explicit knowledge in any of the experiments, and in none of the experiments did the pattern of results change when subjects with explicit knowledge were removed from the analysis. Explicit knowledge will not be discussed further.

The results of this experiment indicate that there is no adverse effect of a variable RSI on learning. One might propose that the effect was not observed because of limited power, but Stadler (1995, Experiment 1) reported a substantial effect $(f=.61)$ when subjects who saw a variable RSI were compared with subjects who saw a consistent RSI (but saw letters or heard tones that they were told to ignore, as a control for another condition). If we assume an effect size comparable to that which Stadler observed, power in the present experiment is .99 .

As noted above, subjects trained with consistent RSIs saw only the longest RSI $(850 \mathrm{msec})$ because if subjects in the random RSI condition showed impaired learning, we wanted to be sure that this impairment was due to the inconsistency of the RSI, not because they saw long RSIs on one third of the trials. But given that the random-RSI subjects appeared to show normal learning, one might ask whether the consistent RSI group would have shown still better learning had the consistent RSI been 450 instead of 850 msec.

To ensure that this confound was not the reason why the two groups of subjects showed equivalent learning, a second experiment was conducted in which the average RSI was the same in the random and in the consistent RSI conditions.

\section{EXPERIMENT 2}

\section{Method}

Subjects. Twenty-four undergraduates at the University of Virginia (4 male) participated in return for course credit in an introductory psychology course.

Stimuli and Apparatus. These were identical to those in Experiment 1.

Procedure. This was identical to that of Experiment 1, except that the RSI in the consistent condition was $450 \mathrm{msec}$. As in Experiment 1 , the RSIs in the random condition were 50,450 , and $850 \mathrm{msec}$ 


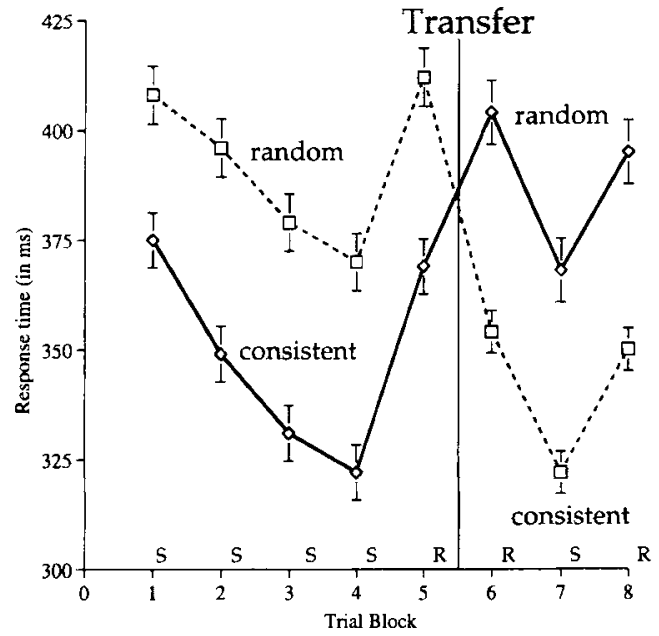

Figure 2. Mean response times across trial blocks as a function of RSI in Experiment 2. Error bars are standard errors, calculated within subjects.

\section{Results and Discussion}

There was a reliable effect of accuracy during training blocks $\left[F(4,88)=6.0, M S_{\mathrm{e}}=2.7, p<.001\right]$ and test blocks $\left[F(2,44)=3.8, M S_{\mathrm{e}}=3.4, p<.05\right]$. These effects were due to consistently higher accuracy $(95.8 \%$ or higher) on the sequenced blocks and lower accuracy (95.0\% or lower) on the random blocks. There was no group effect or interaction for accuracy on either training or test trials (all $F_{\mathrm{s}}<1.5$, all $p \mathrm{~s}>.20$ ).

RTs for each subject were summarized as they were in Experiment $\mathrm{I}$, and the summarized means appear in Figure 2. An analysis of variance (ANOVA) of the sequenced training blocks showed that subjects in the consistent RSI condition responded more slowly than the random RSI subjects $\left[F(1,22)=6.03, M S_{\mathrm{e}}=7,528, p<\right.$ $.05]$, and there was a reliable change in RTs across blocks $\left[F(3,66)=22.35, M S_{\mathrm{e}}=440, p<.0001\right]$. Most importantly, there was no interaction of these effects $\left[F(3,66)=4.54, M S_{\mathrm{e}}=440, p>.20\right]$.

The difference in RTs for trial Blocks 4 and 5 was taken as a second measure of sequence learning. That comparison showed that subjects in the consistent and the random RSI conditions both learned reliably $[F(1,22)=$ $\left.22.02, M S_{\mathrm{e}}=641, p<.001\right]$, and both groups showed equivalent learning $\left[F(1,22)=0.02, M S_{\mathrm{e}}=641, p>.20\right]$.

At transfer, the learning measure was the difference of the sequenced block and the mean of the two random blocks. This measure was significantly different from zero, indicating that subjects showed sequence knowledge at transfer $[t(23)=6.1 ; M=37.3, S E M=6.1]$, and this knowledge did not differ between conditions $[F(1,22)$ $\left.=0.02, M S_{\mathrm{e}}=922, p>.20\right]$. Each group showed reliable learning when analyzed independently (both $t \mathrm{~s}>$ $3.5, p s<.05)$.

Thus, Experiment 2 replicated Experiment 1, with a consistent RSI that matched the mean RSI of the random condition. We propose that the variability of the RSI is not important to learning, but the length of the RSI may be. We turn now to manipulations of RSI length.

\section{EXPERIMENT 3}

The purpose of this experiment was to test whether a long RSI would inhibit learning. The design was identical to that of Experiment 1, except that the independent variable was the length of the RSI, not its consistency.

\section{Method}

Subjects. Thirty-six undergraduates at the University of Virginia (14 male) participated in return for course credit in an introductory psychology course.

Stimuli and Apparatus. These were identical to those of Experiment 1.

Procedure. This was identical to that of Experiment 1, with one exception. The independent variable was not the consistency of the RSI but its length. During the training phase (Blocks 1-5), half of the subjects saw stimuli presented with a short RSI ( $500 \mathrm{msec}$ ), and half saw stimuli with a long RSI ( $1,500 \mathrm{msec})$. During the transfer phase (Blocks 6-9), the RSIs were switched for the two groups, so that subjects who saw short RSIs during training now saw long RSIs, and vice versa. These two conditions are referred to as short-long and long-short, reflecting the RSIs at training and transfer. The order of sequenced and random trial blocks was the same as in Experiment 1 .

\section{Results and Discussion}

As in Experiment 1, there were no reliable effects of accuracy, and accuracy was consistently high (means of $95 \%-97 \%$ within blocks and conditions) and will not be discussed further.

RTs for each subject were summarized as they were in Experiment 1 , and the summarized means appear in Figure 3. An ANOVA of the sequenced training blocks showed that subjects in the long-short condition responded more slowly during training than the subjects in the short-long condition $\left[F(1,34)=4.44, M S_{\mathrm{e}}=9,778\right.$,

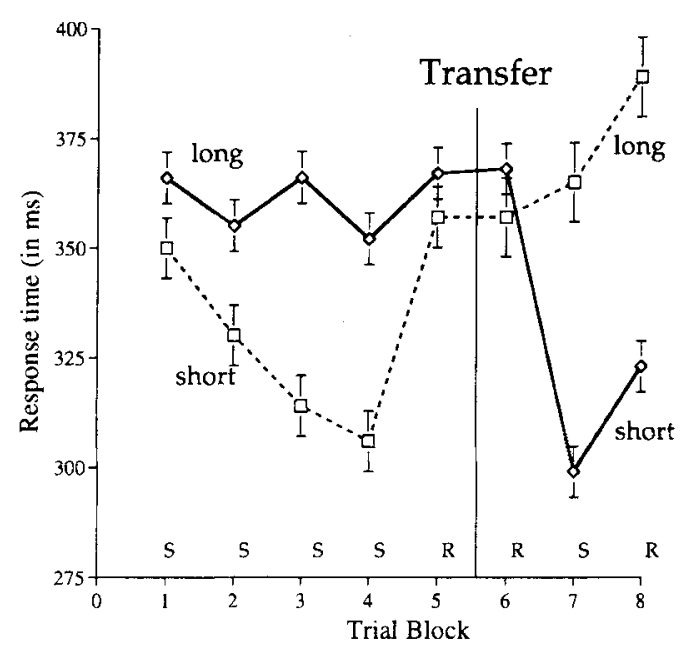

Figure 3. Mean response times across trial blocks as a function of RSI in Experiment 3. Error bars are standard errors, calculated within subjects. 
$p<.05$ ], and there was a reliable change in RTs across blocks $\left[F(3,102)=8.98, M S_{\mathrm{e}}=577, p<.05\right]$. Most importantly, there was an interaction of these effects $[F(3,102)$ $\left.=4.54, M S_{\mathrm{e}}=577, p<.05\right]$. As is apparent from Figure 3 , this interaction is due to RTs' decreasing more quickly in the short-long condition than in the longshort condition.

The difference in RTs for Trial Blocks 4 and 5 was taken as a second measure of sequence learning. That comparison showed that subjects in the short-long condition learned reliably more than those in the long-short [51.1 vs. $\left.18.3 \mathrm{msec} ; F(1,34)=5.3, M S_{\mathrm{e}}=2,288, p<.05\right]$.

At transfer, the learning measure showed a reliable difference $\left[F(1,34)=10.6, M S_{\mathrm{e}}=1,292, p<.05\right]$, with subjects in the short-long condition showing no learning $[t(17)=0.8, p>.2]$ and those in the long-short condition showing reliable learning $[t(17)=6.3, p<.05]$. Notably, with the switching of RSI conditions, the group showing learning was switched. Subjects trained at $500 \mathrm{msec}$ showed no evidence of sequence knowledge once transferred to $1,500 \mathrm{msec}$, and subjects trained at $1,500 \mathrm{msec}$ who seemed not to learn the sequence did show evidence of learning.

Inspection of Figure 3 makes it clear, however, that this planned learning measure may have been affected too much by Block 6 , the first random block at transfer. Suppose that RTs during that block should not have been analyzed because subjects were likely adjusting to the new RSI. Rather, the learning measure should simply have been the difference between Blocks 7 and 8--the sequenced and final random blocks. In that case, both conditions showed learning $\left[F(1,34)=19.5, M S_{\mathrm{e}}=526\right.$, $p<.05]$, and the learning did not differ $[F(1,34)=0.01$, $\left.M S_{\mathrm{e}}=526, p>.20\right]$. Furthermore, both groups showed reliable learning when analyzed alone $(t \mathrm{~s}>2.7, p \mathrm{~s}<.01)$.

The training phase of this experiment replicated Frensch and Miner's (1994) results. Subjects trained with a long RSI showed impaired sequence learning in comparison with subjects trained with a short RSI. The transfer phase, however, revealed that subjects who apparently had not learned the sequence during the training phase showed that they in fact had sequence knowledge once transferred to the short RSI. It appears, then, that subjects' apparent failure to learn the sequence during training with the $1,500-\mathrm{msec}$ RSI was not really a failure to learn; the long RSI affected performance, not learning.

It would appear to be possible that subjects trained with the long RSI and then transferred to the short RSI were not showing knowledge that they had already acquired; rather, they knew nothing about the sequence when transferred to the short RSI but learned the sequence within the single sequenced transfer block. We think this very unlikely, because of the results from a control condition designed to test a similar question for another experiment. In that condition, subjects watched (without responding to) random stimuli for five blocks of trials and then responded to four blocks of trials. The third trial block of stimuli was sequenced, and the RSI was $300 \mathrm{msec}$ throughout the experiment. The subjects showed absolutely no learning of the sequence from the single sequenced trial block (Willingham, 1997). Although the conditions were somewhat different, it appears unlikely that the learning observed in Experiment 3 here was due simply to rapid learning of the sequence.

Another question is whether subjects trained at the short RSI would continue to show sequence knowledge once transferred to the long RSI. The data were ambiguous on this question. Subjects showed no advantage when the sequence was reintroduced at Block 7, but their RTs did increase upon the reintroduction of the random stimuli in Block 8.

The transfer RTs of the subjects switched from short to long RSIs increased from block to block. Why should these RTs have increased? This pattern matches spontaneous comments from subjects in this condition upon completion of the experiment. Many remarked that the task became very boring; some delivered these comments in frank irritation. We hypothesized that the increasing RTs were due to a lack of effort on the part of these subjects as they became increasingly bored and frustrated by the task; therefore, the increase in RTs from Block 7 to 8 was not indicative of learning, but of boredom.

If these effects were indeed present, they obviously represented a confound that could obscure the true data pattern. Experiments 4 and 5 were attempts to motivate these subjects so as to reduce or eliminate such effects.

\section{EXPERIMENT 4}

\section{Method}

Subjects. Thirty-four undergraduates at the University of Virginia (13 male) participated in return for course credit in an introductory psychology course.

Stimuli and Apparatus. These were identical to those of the previous experiments.

Procedure. The procedure was identical to that of Experiment 3, except for the following changes. At the end of the training phase, all subjects took a 2-min break, during which they were encouraged to get a drink of water, take a brief walk, and so on. All subjects were also told that the experimenters were particularly interested in subjects trying hard in the last phase of the experiment and were therefore offering a small reward for subjects whose RTs were in the top 25th percentile for the experiment. The rewards were the subjects' choice of a candy bar or soda. All subjects were in fact offered the reward at the end of the experiment.

Another change in the procedure was inadvertent and was brought to our attention only after the experiment's completion. The computer program controlling the experiment typically displayed a message on the screen at the end of the training phase instructing the subject to notify the experimenter that the first phase of the experiment was completed. An error in that program prevented that message from being displayed, so the technician testing subjects asked subjects to count the number of trial blocks, and to find him after five blocks had been completed. The possible significance of this change is discussed below. 


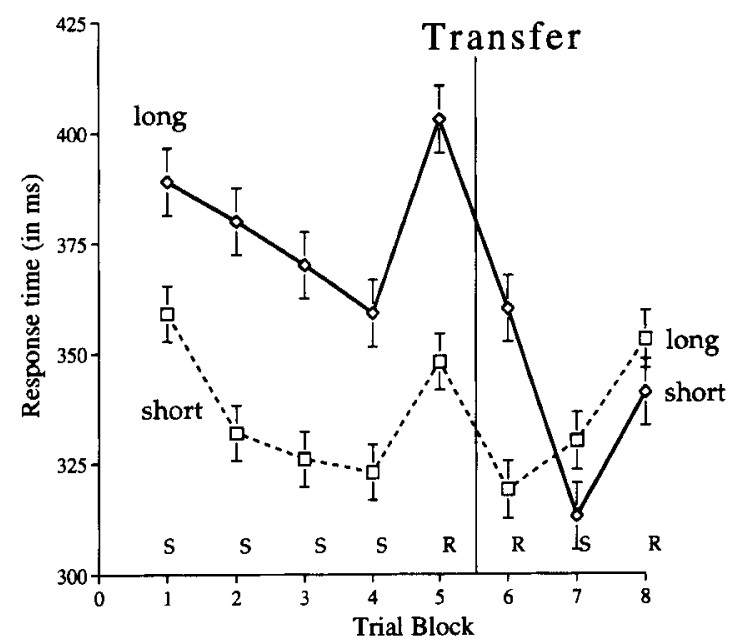

Figure 4. Mean response times across trial blocks as a function of RSI in Experiment 4. Error bars are standard errors, calculated within subjects.

\section{Results and Discussion}

As in previous experiments, accuracy was uniformly high (95\%-97\% within blocks and conditions) and will not be discussed further.

RTs for each subject were summarized as in the previous experiments, and the mean RTs for each condition across trial blocks are shown in Figure 4. An ANOVA of the sequenced training blocks showed that subjects in the long-short condition responded more slowly during training than those in the short-long $[F(1,32)=4.73$, $\left.M S_{\mathrm{e}}=11,119, p<.05\right]$, and there was a reliable change in RTs across blocks $\left[F(3,96)=8.97, M S_{\mathrm{e}}=754, p<\right.$ $.05]$. These results replicate those of Experiment 3. Unlike in Experiment 3, however, there was no difference in learning between the short-long and long-short conditions $\left[F(3,96)=0.75, M S_{\mathrm{e}}=754, p>.20\right]$, indicating that subjects in both conditions learned the sequence. Indeed, separate analyses of each group showed reliable learning $(F \mathrm{~s}>2.8, p \mathrm{~s}<.05)$.

The difference in RTs for Trial Blocks 4 and 5 is the second measure of learning, and that too indicates that both conditions learned the sequence. The difference score for both conditions was reliable (both $t \mathrm{~s}>3.00$, $p \mathrm{~s}<.01)$. Although the learning score for the long-short condition was larger than that for the short-long condition, it was not reliably larger $\left[F(1,32)=1.4, M S_{\mathrm{e}}=\right.$ $2,273, p>.20]$.

The pattern of RTs at transfer replicated that in Experiment 3 . The use of the difference score between the two random blocks and the sequenced block as the dependent measure indicated different learning between conditions $\left[F(1,32)=6.5, M S_{\mathrm{e}}=1,332, p<.05\right.$, with the short-long condition showing no learning $[t(16)=$ $0.7, p>.20$ ] and the long short condition showing reliable learning $[t(16)=4.0, p<.05]$. But, as in Experiment 3 , the real difference between conditions was in the first random block at transfer. If one takes the difference between Blocks 7 and 8 as the dependent measure, both conditions showed evidence of learning $[F(1,32)=14.1$, $\left.M S_{\mathrm{e}}=762, p<.05\right]$, and the learning did not differ $\left[F(1,32)=0.20, M S_{\mathrm{e}}=762, p>.20\right]$. Separate analyses of each group showed reliable learning (both $t \mathrm{~s}>2.4$, $p$ s <.05).

This experiment replicated the main aspects of Experiment 3 . Two results are particularly noteworthy. The reward seems to have had little effect on RTs. It is possible that the incentive was not great enough, or that motivation was not the problem in the first place; but, for whatever reason, subjects who were transferred from the 500-msec RSI to the 1,500 -msec RSI showed progressively increasing RTs at transfer.

The second difference in the results of this study was that subjects in the long-short condition showed normal learning during training. This result is in direct opposition to the results of Experiment 3. A possible explanation may be found in the change in instructions noted in the Method section. In Experiment 4, subjects were asked to count the number of trial blocks that they completed during transfer. It seems plausible that knowing how many more trials they had to complete was motivating for subjects, so that the subjects in Experiment 4 tried harder than the subjects in Experiment 3 and hence showed a performance improvement.

We examined that hypothesis in Experiment 5 by changing the instructions.

\section{EXPERIMENT 5}

The purpose of this experiment was to replicate Experiment 3 without the confounding effect that knowledge of the number of trial blocks might have on performance.

\section{Method}

Subjects. Thirty-five undergraduates at the University of Virginia (17 male) participated in return for course credit in an introductory psychology course.

Stimuli and Apparatus. These were identical to those in the previous experiments.

Procedure. This was identical to that of Experiment 4, with the following changes. The subjects were not told to count trial blocks during training, nor were they told the total number of trial blocks that they would perform; a message appeared on the computer screen at the end of training asking them to get the experimenter. The experimenter then told subjects that the timing of the stimuli would change. If knowing the number of trial blocks left in the experiment motivated improved performance, it would be desirable to apply that manipulation at transfer, where motivation might be a problem. Therefore, as the transfer task started, subjects were told that they would complete three more trial blocks and that they should summon the experimenter at the blocks' conclusion.

\section{Results and Discussion}

All of the results from Experiment 4 were replicated in Experiment 5. The mean RTs for each block across conditions are shown in Figure 5.

As in previous experiments, accuracy was uniformly high (95\%-98\% within blocks and conditions) and will not be discussed further. 


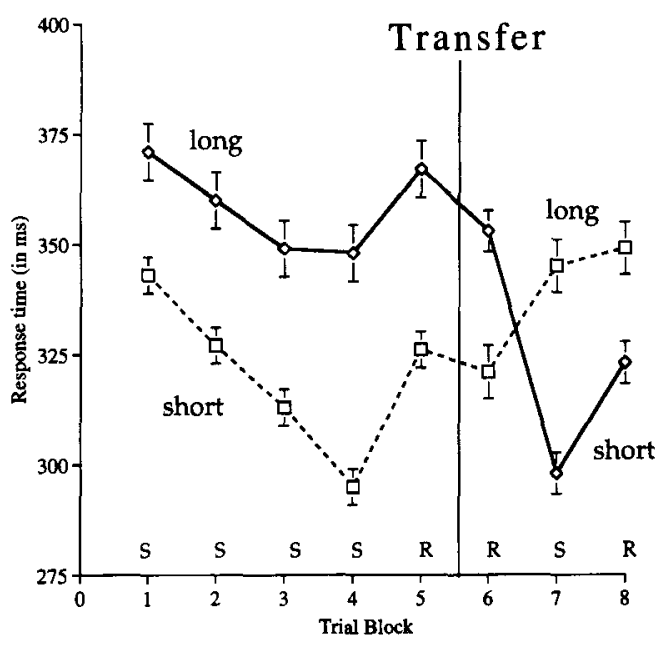

Figure 5. Mean response times across trial blocks as a function of RSI in Experiment 5. Error bars are standard errors, calculated within subjects.

An ANOVA of the sequenced training blocks showed that subjects in the long-short condition responded more slowly than those in the short-long during training $\left[F(1,33)=5.48, M S_{\mathrm{e}}=8,908, p<.05\right]$, and there was a reliable change in RTs across blocks $[F(3,99)=19.2$, $\left.M S_{\mathrm{e}}=426, p<.05\right]$. There was a marginal difference in learning between the short-long and long-short conditions $\left[F(3,99)=2.40, M S_{\mathrm{e}}=426, p=.07\right]$. Both groups showed learning when analyzed independently $(F \mathrm{~s}>$ $3.0, p \mathrm{~s}<.05$ ).

The difference in RTs for Trial Blocks 4 and 5 indicates that both conditions learned the sequence equally well. The difference score for both conditions was reliable (both $t \mathrm{~s}>1.8$ ). The learning score for the the shortlong condition was larger than that of the long-short condition, but not reliably so $\left[F(1,33)=1.09, M S_{\mathrm{e}}=\right.$ $1,202, p>.20]$.

If the difference between the sequenced block and the two random blocks is used as the dependent measure, there was a difference between conditions at transfer $\left[F(1,33)=26.86, M S_{\mathrm{e}}=788, p<.05\right]$. Subjects seeing long RSIs at transfer showed no evidence of learning $(t=-1.1, p>.20)$, whereas subjects seeing short RSIs at transfer did show evidence of learning $(t=11.3, p<$ .001 ). If one simply uses the difference between the sequenced block and the last random block as the dependent measure, this basic result was replicated: the long-short subjects showed reliable learning $(t=5.0$, $p<.001)$, whereas the short-long subjects did not $(t=0.7, p>.20)$. This difference in learning was reliable $\left[F(1,33)=6.11, M S_{\mathrm{e}}=295, p<.05\right]$. The latter result differs from Experiments 3 and 4, and appears to have been due to the short-long subjects' responding more quickly on the final block; that is, their RTs stopped increasing and remained at the same level as in Block 7.
Experiment 5 replicated Experiment 4 in the main. Thus, the fact that the long-short subjects showed learning during training appears not to have been due to their knowing the number of trial blocks in the training phase.

The transfer results did appear to be slightly different in Experiment 5; short-long subjects showed no evidence of transfer according to either learning measure, whereas the data were somewhat more ambiguous in prior experiments. This result may have been due to our instruction to subjects to count the trial blocks at transfer, but if so, it only influenced RTs on the final trial block; subjects' RTs still increased dramatically from the sixth to the seventh trial block.

In Experiment 6, we abandoned the apparently unapproachable goal of getting subjects who were transferred to the $1,500-\mathrm{msec}$ RSI to respond quickly. Experiment 6 was designed to control this problem, not to eliminate it. The subjects were trained at long or short RSIs and then their RSI conditions were switched at transfer, as before. In this experiment, half of the subjects saw only random stimuli at transfer, so the effect of changing the RSI could be evaluated independently of the effect of the sequence. Because Frensch and Miner (1994) argued that $1,500 \mathrm{msec}$ is about the shortest RSI that will disrupt learning, we also lengthened the long RSI to $2,000 \mathrm{msec}$ in an effort to maximize its effect.

\section{EXPERIMENT 6}

\section{Method}

Subjects. Sixty-four undergraduates at the University of Virginia (29 male) participated in return for course credit in an introductory psychology course.

Stimuli and Apparatus. These were identical to those in the previous experiments.

Procedure. The procedure was identical to that in Experiment 3, except for two changes. First, the long RSI was 2,000 msec instead of 1,500. The short RSI was still $500 \mathrm{msec}$. Second, at transfer, the subjects' RSI conditions, were switched as in the previous experiments. However, half of the subjects in each condition saw only random stimuli at transfer, whereas the other subjects saw the random, sequenced, random set of trial blocks used in previous experiments.

\section{Results and Discussion}

As in the previous experiments, accuracy was uniformly high ( $95 \%-97 \%$ within blocks and conditions) and will not be discussed further.

The subjects in the short-long condition responded more quickly than the long-short subjects $[F(1,60)=$ $\left.6.33, M S_{\mathrm{e}}=7,890, p<.05\right]$, and for some reason this effect was pronounced in the condition that would see the sequence at transfer, but not in the condition that would see only random stimuli at transfer $[F(1,60)=4.12$, $\left.M S_{\mathrm{e}}=7,890, p<.05\right]$. It should be borne in mind that both conditions had been treated identically during training, and that this interaction can only be regarded as having been due to chance. More importantly, all subject groups showed reliable learning of the sequence $[F(3,180)$ $\left.=23.47, M S_{\mathrm{e}}=910, p<.05\right]$, and this learning did not 


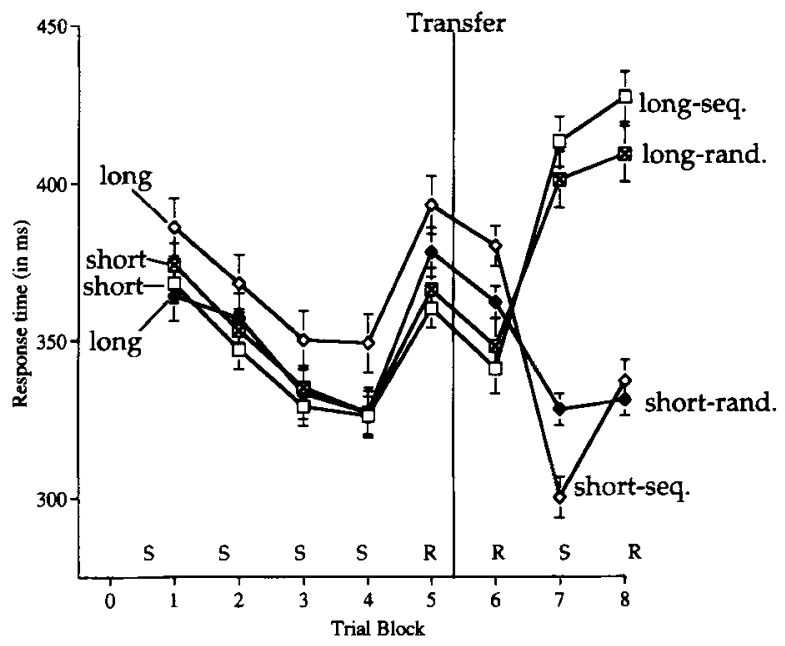

Figure 6. Mean response times as a function of trial blocks, RSI, and stimuli at transfer in Experiment 6. Block 7 stimuli were either sequenced $(S)$ or random $(R)$. Error bars are standard errors, calculated within subjects.

vary among conditions (for all interactions, $F \mathrm{~s}<1.0$ ). All subject groups showed reliable sequence learning when analyzed separately $\left(F_{\mathrm{S}}>4.4, p \mathrm{~s}<.01\right)$.

Using the difference score between Trial Blocks 4 and 5 yielded the same result. All conditions showed reliable learning (all $t \mathrm{~s}>2.9$ ) and no effects of RSI or of random/sequenced stimuli at transfer (all $F \mathrm{~s}<1.1)$.

From the transfer data in Figure 6, it appears that only subjects in the long-short condition showed sequence knowledge during the transfer trials. That impression was borne out by the analyses.

At transfer, half of the subjects received one sequenced block and half did not. The learning measure used in previous experiments (the mean of the first and third transfer block minus the second transfer block) revealed a reliable interaction of RSI and sequenced/random $[F(1,60)$ $\left.=8.07, M S_{\mathrm{e}}=1,116, p<.05\right]$. Planned post hoc contrasts of this interaction showed that short-long subjects who saw the sequence had learning scores no different than subjects seeing only random stimuli $[F(1,30)=$ $\left.0.31, M S_{\mathrm{e}}=1,224, p>.20\right]$. Long-short subjects, however, showed an effect of sequenced/random stimuli $\left[F(1,30)=13.1, M S_{\mathrm{e}}=1,008, p<.05\right]$; in other words, subjects seeing stimuli with short RSIs at transfer showed knowledge of the sequence.

The other measure of learning at transfer was simply the difference score for Blocks 7 and 8 , and the all-random conditions clarified the data pattern obtained in prior experiments. Although the interaction of RSI and sequence/ random was marginal $\left[F(1,60)=3.47, M S_{\mathrm{e}}=1,012\right.$, $p=.07]$, the planned contrasts were conducted, and the results replicated the findings obtained with the other learning measure: seeing the sequence conferred no advantage on the learning score for subjects in the shortlong condition $\left[F(1,30)=0.2, M S_{\mathrm{e}}=1,169, p>.20\right]$, but did for the long-short condition $[F(1,30)=11.5$,
$\left.M S_{\mathrm{e}}=855, p<.05\right]$. Thus it is clear that subjects in the short--long RSI condition did not show any evidence of sequence knowledge at transfer.

This experiment again replicated the finding that subjects did learn the sequence during training with a long RSI, even when the RSI was $2,000 \mathrm{msec}$. It also showed conclusively that subjects who were transferred from the short to the long RSI did not show evidence of having learned, but subjects transferred from long to short RSI did.

\section{GENERAL DISCUSSION}

This set of experiments was motivated by three data sets: Nissen and Bullemer's (1987) report that a tonecounting distractor task disrupted learning, which they interpreted as an effect of attention, and reinterpretations of the tone-counting task as operating on subjects' ability to organize the sequence (Stadler, 1995) or to maintain successive positions in working memory (Frensch \& Miner, 1994). The data reported here are difficult to reconcile with the latter two hypotheses.

Stadler $(1992,1993,1995)$ has proposed that the organization of sequences is critical to the ease with which subjects learn them. He argues that RT reduction in the SRT task occurs to the extent that the same run of trials (e.g., three trials) is practiced more often, but, that for a run to be recognized as having appeared before, it must be grouped in the same way. Pauses inserted between the trials may affect grouping. Attention, Stadler argues, is important for grouping and so affects learning indirectly.

Experiments 1 and 2 show that this is not always true. When inconsistent but short RSIs are used, there is no effect on learning, and subjects continue to show good performance at transfer. It is possible that the RSIs in Experiments 1 and 2 were not discrepant enough; the difference between the longest and shortest RSI that Stadler (1995) used was $1,500 \mathrm{msec}$, whereas in this experiment it was $800 \mathrm{msec}$. This possibility cannot be ruled out and indeed can never be ruled out if consistency of RSI is not to be confounded with absolute length of RSI.

Frensch and Miner (1994) proposed that a consistent, long RSI prevents sequence learning in the SRT task. We have found no evidence to support that contention. Subjects showed learning during the training phase in three of four experiments and showed evidence of learning in all of the transfer phases. But subjects seeing stimuli with a long RSI at transfer (and trained at the short RSI) showed no evidence of learning in all four experiments. Clearly, these subjects knew the sequence-that they failed to show this knowledge must be an effect of performance, not learning.

Our results raise two obvious questions: Why did our results from training differ from Frensch and Miner's (1994), and what is the source of the transfer effect? Frensch and Miner did use a different sequence of stimuli, what Cohen et al. (1990) have called "hybrid" sequences (some stimuli are followed by one stimulus; others are followed by more than one); we used longer "ambiguous" sequences 
(all stimuli can be followed by more than one stimulus). Although it is possible that the lack of learning that they observed was due to this difference, it would be a bit puzzling, since hybrid sequences are usually learned at least as fast as ambiguous sequences.

Our results are not as different from Frensch and Miner's (1994) as they might seem at first. Although Frensch and Miner concluded that subjects at the long RSI showed impaired learning in their second experiment, they found that these subjects in fact showed normal learning on two of the three possible learning measures. Compared with subjects seeing stimuli with a short RSI, these subjects showed a smaller increase in RTs when transferred from the sequenced to the random stimuli, but they showed a normal decrease in RTs during the sequenced training blocks and also showed a normal RT decrement when the sequence was reintroduced. In other words, Frensch and Miner's data, like ours, have elements consistent with impaired learning and consistent with spared learning at long RSIs.

There is no ambiguity about the transfer data from the experiments reported here, however, and therefore we argue that, although the long RSI might sometimes affect subjects' performance, it does not affect learning; subjects consistently show normal learning once they are transferred to a short RSI. But why do subjects not show evidence of learning when transferred from the short to the long RSI?

This effect may have been due to attention. Subjects consistently reported being frustrated and bored when transferred to the slower RSI. When subjects who began the task with the slower RSI were queried, they seldom described such feelings. We do not have formal measures of these reports, but it appears that starting the task with the short RSI led to expectations of a certain minimum level of interest in the task; when the task became more boring, subjects were frustrated. If subjects began the experiment with the long RSI, however, they could not compare it to the short RSI condition.

If this scenario is accurate, it implies that tone counting may also affect performance, not learning. Nissen and Bullemer (1987, Experiment 3) tested this hypothesis but found no support for it; subjects trained with a distractor task showed no knowledge of the sequence when they performed the task without the distractor task. However, a recent report has indicated that subjects who do not show learning with a secondary task do show evidence of sequence knowledge when the secondary task is removed (Frensch, Lin, \& Buchner, in press).

The idea that RSI affects performance but not learning is interesting. Implicit memory is usually thought of as influencing performance automatically, without conscious effort on the part of the subject. Indeed, subjects don't even know that memory is being tested. How, then, could attention be important in the expression of implicit knowledge? Implicit knowledge may not require a conscious decision to be applied, but it does require that conditions in the environment are appropriate; there are conditions in which implicit memory could be applied but is not, and in fact some have argued that the conditions at encoding and retrieval must be quite closely matched for implicit memory to influence performance (N. J. Cohen \& Eichenbaum, 1993; Glisky, Schacter, \& Tulving, 1986). In this sense, the application of implicit memory requires recognition; the system underlying implicit memory performance must recognize that the environmental conditions are right for applying implicit knowledge. This process of recognition may require attention to the environment.

\section{REFERENCES}

Cohen, A., Ivry, R. I., \& KeELE, S. W. (1990). Attention and structure in sequence learning. Journal of Experimental Psychology: Learning, Memory, \& Cognition, 16, 17-30.

Cohen, N. J., \& Eichenbaum, H. (1993). Memory, amnesia, and the hippocampal system. Cambridge, MA: MIT Press.

Curran, T., \& Keele, S. W. (1993). Attentional and nonattentional forms of sequence learning. Journal of Experimental Psychology: Learning, Memory, \& Cognition, 19, 188-202.

FrensCh, P. A., Lin, J., \& Buchner, A. (in press). Learning versus behavioral expression of the learned: The effects of a secondary tonecounting task on implicit learning in the serial reaction time task. Psychological Research.

FRENSCH, P. A., \& MiNER, C. S. (1994). Effects of presentation rate and individual differences in short-term memory capacity on an indirect measure of serial learning. Memory \& Cognition, 22, 95-110.

Glisky, E. L., Schacter, D. L., \& Tulving, E. (1986). Computer learning by memory impaired patients: Acquisition and retention of complex knowledge. Neuropsychologia, 24, 313-328.

GlucksBerG, S., \& CowAN, G. N. (1970). Memory for nonattended auditory material. Cognitive Psychology, 1, 149-156.

GRAF, P., \& SCHACTER, D. L. (1985). Implicit and explicit memory for new associations in normal and amnesic subjects. Journal of Experimental Psychology: Learning, Memory, \& Cognition, 11, 501-518.

Keele, S. W., \& Jennings, P. J. (1992). Attention in the representation of sequence: Experiment and theory. Human Movement Science, 11, 125-138.

Nissen, M. J., \& Bullemer, P. (1987). Attentional requirements of learning: Evidence from performance measures. Cognitive Psychology, 19, 1-32.

Schacter, D. L., Chiu, C. Y. P., \& OChSNer, K. N. (1993). Implicit memory: A selective review. Annual Review of Neuroscience, 16, 159182.

Squire, L. R., Knowlton, B., \& Musen, G. (1993). The structure and organization of memory. Annual Review of Psychology, 44, 453495.

STADLER, M. A. (1992). Statistical structure and implicit serial learning. Journal of Experimental Psychology: Learning, Memory, \& Cognition, 18, 318-327.

STADLER, M. A. (1993). Implicit serial learning: Questions inspired by Hebb (1961). Memory \& Cognition, 21, 819-827.

STADLER, M. A. (1995). The role of attention in implicit learning. Journal of Experimental Psychology: Learning, Memory, \& Cognition, 21, 674-685.

Willingham, D. B., Nissen, M. J., \& Bullemer, P. (1989). On the development of procedural knowledge. Journal of Experimental Psychology: Learning, Memory, \& Cognition, 15, 1047-1060.

WillinghaM, D. B. (1997). Implicit motor sequence learning is not purely perceptual. Manuscript submitted for publication.

(Manuscript received June 19, 1995 revision accepted for publication July $30,1996$. 